\title{
THE THEORY OF EXTENDED ATMOSPHERES
}

\author{
L. H. Aller
}

It is pretentious to speak of a theory of extended stellar envelopes since except in a few limiting cases all one can do is to propose hypotheses that may be helpful in assessing some special set of observations. The principle types of object are: (I) late-type giants and supergiants including objects such as Cepheids, irregular and long-period variables; (2) circumstellar envelopes around binary stars; (3) novae; (4) combination variables; (5) Be, Of, and Wolf-Rayet stars; (6) stars with extremely tenuous outer envelopes such as planetaries; and (7) stars in the process of formation.

Theoretical analyses of extended envelopes are based on some sort of an assumed model. A preliminary geometrical picture is established from qualitative and quantitative data concerning the general level of excitation of the spectrum, line shapes and displacements. The geometrical model is linked with a physical model by adopting mechanisims of energy input and dissipation. In most models the hydrodynamical and kinematical aspects of the problem must be considered. Magnetic fields are almost surely important in orderly expanding envelopes such as planetary nebulae. So far only rather primitive models and theories have been discussed, particularly for the combination variables where departures from thermodynamic equilibrium appear to be large.

\section{9b. SOUS-COMMISSION DES ETALONS D'INTENSITE DE RAIES}

\section{Report of Meeting, 9 August 196 r}

President: K. O. Wright.

SECRETARY: J. Houtgast.

The President in his introductory remarks noted that, although this would be the last meeting as a Sub-Commission of Commission 29, the need for inter-observatory comparisons of line intensities seemed to be as great as ever. In the discussion of the Draft Report, Miss Underhill thought that some of the lower values of equivalent widths obtained by photoelectric spectral scanning might be the result of the relatively short scan in wave-length and the influence of absorption lines on the continuum.

Several short communications were presented:

I. F. B. Oke discussed the Photo-electric Scanning of Stellar Spectra at Mount Wilson Observatory. No results in addition to those listed in the Draft Report have yet been obtained, but it is possible that a dispersion of $\mathbf{I} \cdot \mathrm{I} \AA / \mathrm{mm}$ may be used in the future.

2. N. F. Woalf commented on the Fabry-Perot Monochromator that has been described by Geake, Ring and Woolf (M.N. r19, 616, 1959) and that is now being tested at the Lick Observatory. Although no exact quantitative results have yet been obtained, the method appears to be very promising, especially for sharp interstellar lines.

3. F. Houtgast described the Photo-electric Scanning of Solar Lines at the Utrecht Observatory. The influence of spectral resolution on the equivalent widths of $\mathrm{H} \delta$ and $\mathrm{Ca} \mathrm{I}$, $\lambda_{4227}$ were investigated and appeared to be small. It was thought useful to continue this work and to include the effects that dispersion may have on equivalent widths when determined photo-electrically. 
4. K. O. Wright described results obtained from a Photographic Comparison of the Kienle Step-Filter and the Victoria Rotating Sector. When care is taken to ensure uniform illumination over the field, the calibration curves are found to be identical within the errors of measurement. He also discussed a table of equivalent widths of lines in the spectrum of Procyon; pre-I $95^{\circ}$ observations showed large divergences especially for weak lines, but for later observations obtained with high dispersion the scatter is decreased appreciably.

The President had brought the step-filters of Kienle and Minnaert that had been calibrated by Kienle, and they were on display at the meeting. They have been used to check the calibration procedures at Victoria, Saltsjöbaden and Asiago. Taffara had sent the results of his comparison of the Asiago exponential diaphragm and edge with the Kienle step-filter.

In his concluding remarks the President commented that it seems desirable that there should be some organized group to maintain the interest in co-operative intensity measurements and in calibration problems. He suggested that the Sub-Commission should continue as a Committee of Commission 29. This was approved by all members present (and later by Commission 29).

\section{9c. SOUS-COMMISSION DES CLASSIFICATIONS STELLAIRES}

President: W. P. Bidelman.

\section{Report of Meetings}

Acting Secretary: P. J. Treanor.

\section{First meeting, 17 August r96r}

The President's Report was adopted in the form in which it appears in Vol. XI A of the Transactions, the only significant change from the Draft Report occurring in Appendix II, which was slightly modified at the request of the authors.

W. W. Morgan and H. A. Abt reported on progress on the new Spectral Atlas, for which the plate material, secured on Kitt Peak, is approximately $85 \%$ complete. The atlas is being prepared from grating spectrograms of dispersion $125 \AA / \mathrm{mm}$ which extend to the neighborhood of $\lambda_{3500}$ for early-type stars, but its final form is not yet definite. The atlas will not be available for at least another year.

A. Slettebak reported that the funds allocated three years ago to the Perkins Observatory to support a spectroscopic information center there had been returned to the IAU, because the requisite clerical help was not available. The President noted that an information file on all stars for which luminosity determinations (objective-prism or slit) or remarks concerning spectral peculiarities are available (some 15000 stars) is currently being prepared at the Lick Observatory. Information from this file can be supplied on request; the catalogue uses 1900.0 co-ordinates. C. Jaschek has a similar catalogue for southern objects. The President expressed the opinion that the publication of a complete list of stars that possess two-dimensional classifications is not desirable at the present time, but suggested that any future edition of the Yale Catalogue of Bright Stars or other similar catalogue should contain such data.

K. G. Henize discussed his southern-hemisphere objective-prism classification work on carbon and S stars, and his survey of emission-line objects, while N. G. Roman reported on her unpublished slit-spectra classifications of some 600 objects in various Selected Areas. W. W. Morgan announced that he plans to continue work on the classification of $O B$ stars 